\title{
Investigación e Innovación desde los Centros de Apoyo y Desarrollo Educativo Profesional una Perspectiva Intercultural
}

\author{
Research and Innovation from the Centers of Support and Professional Educational \\ Development an Intercultural Perspective
}

William Oswaldo Flores López ${ }^{1}$

\section{Resumen}

La presente comunicación describe que los Centros de Apoyo y Desarrollo Educativo Profesional promueven la investigación e innovación con una perspectiva intercultural. Es decir, tratan de fomentar la investigación e innovación didáctica, pedagógica y en la generación de nuevas tecnologías para el aprendizaje a partir de un modelo de investigación propia que reconoce la diversidad, multiculturalidad y multilenguaje de las poblaciones enfocada en las soluciones en y para la comunidad. Además, los Centros de Apoyo y Desarrollo Educativo Profesional se caracterizan por la cooperación intra e interinstitucional en la transferencia de buenas prácticas que apoyan, cultivan, adaptan, comunican, innovan y acogen a la comunidad universitaria.

Palabras clave: Investigación, Innovación, Educación, Interculturalidad

\section{Abstract}

This communication describes that the Professional Education Support and Development Centers promote research and innovation with an intercultural perspective. That is to say, they try to promote didactic and pedagogical research and innovation and the generation of new technologies for learning from their own research model that recognizes the diversity, multiculturalism and multilanguage of populations focused on solutions in and for community. In addition, the Professional Education Support and Development Centers are characterized by intra- and interinstitutional cooperation in the transfer of good practices that support, cultivate, adapt, communicate, innovate and welcome the university community.

Key Words: Research, Innovation, Education, Interculturality

1 Doctor en Educación. Profesor Investigador de la Universidad de las Regiones Autónomas de la Costa Caribe Nicaragüense. Correo: william.flores@uraccan.edu.ni (1): http://orcid.org/oooo-ooo2-1016-1620

Recibido: 15/04/2018 Aprobado: 01/o9/2018 


\section{REFLEXIONES EDUCATIVAS}

\section{Introducción}

La investigación e innovación es necesaria en los Centros de Apoyo y Desarrollo Educativo Profesional (CADEP) porque contribuiría al objetivo de desarrollo sostenible sobre educación de calidad, que trata de garantizar una educación inclusiva, equitativa y de calidad y promover oportunidades de aprendizaje durante toda la vida (CEPAL, 2016). Además, se asevera en el informe de resultados del Tercer Estudio Regional Comparativo y Explicativo, que el logro de dicho objetivo "es la base para mejorar la vida de las personas y el desarrollo sostenible" (UNESCO, 2015, p. 8). De forma explicita, se hace en la Declaración de Incheon (2016): "nuestra visión es transformar las vidas mediante la educación, reconociendo el importante papel que desempeña la educación como motor principal del desarrollo y para la consecución de los objetivos de desarrollo sostenibles propuestos" (UNESCO, 2016, p. 7).

La Agenda 2030 se plantea siete metas en referencia con el objetivo 4, y señala la existencia de tres formas de ejecución para el alcance de las misma: generar entornos de aprendizaje eficaces; incrementar sustancialmente el número de becas; aumentar sustancialmente el número de docentes calificados. En este sentido, la Conferencia Regional de Educación Superior 2018, se plantea que la investigación científica, tecnológica y la innovación es el motor del desarrollo humano, social y económico de América Latina y el Caribe, así mismo, se declaró que el conocimiento es un derecho humano universal, un bien público social y común para la soberanía, buen vivir y emancipación de nuestros pueblos, y para la construcción de la ciudadanía latinoamericana y caribeña (UNESCO, 2017).

Así, pues, los Centros de Apoyo y Desarrollo Educativo Profesional tratan de contribuir a la educación a lo largo de toda la vida a través de la investigación e innovación educativa para la búsqueda de soluciones a las problemáticas que afectan a la Educación Superior de América Latina y el Caribe relacionada con: la deserción estudiantil por factores emotivos, académicos, de marginación cultural económica o social, o por discapacidad; la falta de recursos didácticos en profesores universitarios para responder a exigencias de estudiantes en situación de vulnerabilidad; y las brechas de comunicación y cooperación entre la comunidad universitaria que impiden gestar acciones colectivas para enfrentar problemas transversales vinculados al acceso y permanencia exitosa en la universidad (ACACIA, 2015; Flores et al., 2016).

\section{Los Centros de Apoyo y Desarrollo Educativo Profesional}

Los Centros de Apoyo y Desarrollo Educativo Profesional (CADEP) tienen como objetivo apoyar, cultivar, adaptar comunicar, innovar y acoger a la comunidad universitaria en su conjunto y a cada persona en particular, con el fin de favorecer el fomento profesional de todos los miembros de la institución de educación superior; propiciando la disminución de la deserción estudiantil; la modernización de las estructuras organizativas 
y para garantizar el respeto por el otro y sus diferencias, a partir de la cooperación intra e interinstitucional en la producción y desarrollo de recursos didácticos y tecnológicos accesibles, y en la generación de estrategias de fomento socioafectivo en atención a las poblaciones en riesgo de exclusión universitaria (Rodríguez, León \& Gutiérrez y Restrepo, 2017).

En el contexto de la Universidad de las Regiones Autónomas de la Costa Caribe Nicaragüense, el CADEP se fundamenta en la interculturalidad, que constituye un eje transversal en todo el quehacer de la Universidad. En lo académico, ésta debe permitir que docentes y estudiantes establezcan una relación horizontal con los comunitarios y las comunitarias. A la vez, promueve la relación de los saberes endógenos o tradicionales con los conocimientos exógenos u occidentales, permitiendo valorar las costumbres y los saberes propios, así como crear y recrear nuevos conocimientos relevantes al contexto socio-cultural. En los programas curriculares, proyectos de investigación y extensión social comunitaria, la interculturalidad permite a la vez que la población multiétnica, revitalice sus culturas, redescubra y promueva sus valores, sistematice sus saberes, conocimientos y asuma el protagonismo de su desarrollo y el Buen Vivir comunitario. Se trata de un concepto orientado a la construcción de una ciudadanía intercultural, es decir, una ciudadanía que practica el respeto, la tolerancia, la inclusión, la igualdad y equidad entre hombres y mujeres de todos los pueblos y comunidades étnicas (URACCAN, 2018).

\subsection{La Investigación desde los Centros de Apoyo y Desarrollo Educativo Profesional}

La investigación en los Centros de Apoyo y Desarrollo Educativo Profesional es asumida como un proceso participativo con perspectiva intercultural de género, que genera nuevos conocimientos, capacidades e innovaciones, revaloriza las prácticas históricas y saberes locales de los pueblos indígenas, afrodescendientes, mestizo y comunidades étnicas, en la búsqueda del Buen Vivir y la construcción de ciudadanías interculturales (URACCAN, 2017, pp. 5-6). Desde esta perspectiva, los CADEP promueven espacios de creación y recreación de conocimientos, saberes y prácticas en el ámbito educativo, concretamente en investigar proceso de enseñanza y aprendizajes mediante el diálogo y la reflexión para la producción y desarrollo de recursos didácticos y tecnológicos, y en la generación de estrategias de fomento socioafectivo a poblaciones en riesgo de exclusión universitaria. 


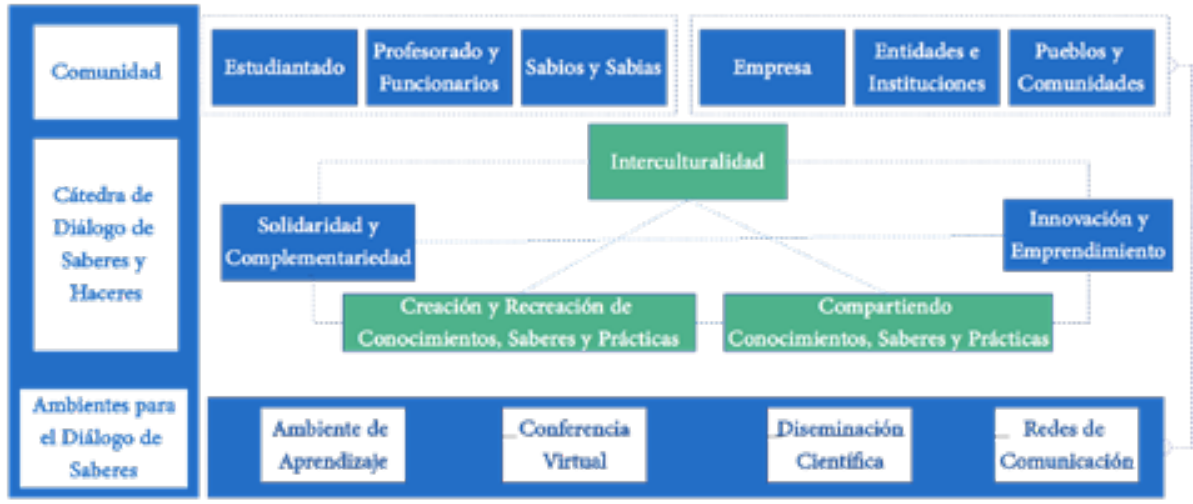

Figura 1: Investigación e Innovación de los Centros de Apoyo y Desarrollo Educativo Profesional

La investigación en los Centros de Apoyo y Desarrollo Educativo Profesional inicia con el reconocimiento de las situaciones problemáticas del contexto educativo hasta la reflexión y generación de nuevo conocimiento que logran los mejores resultados en la práctica de forma eficiente y equitativa.

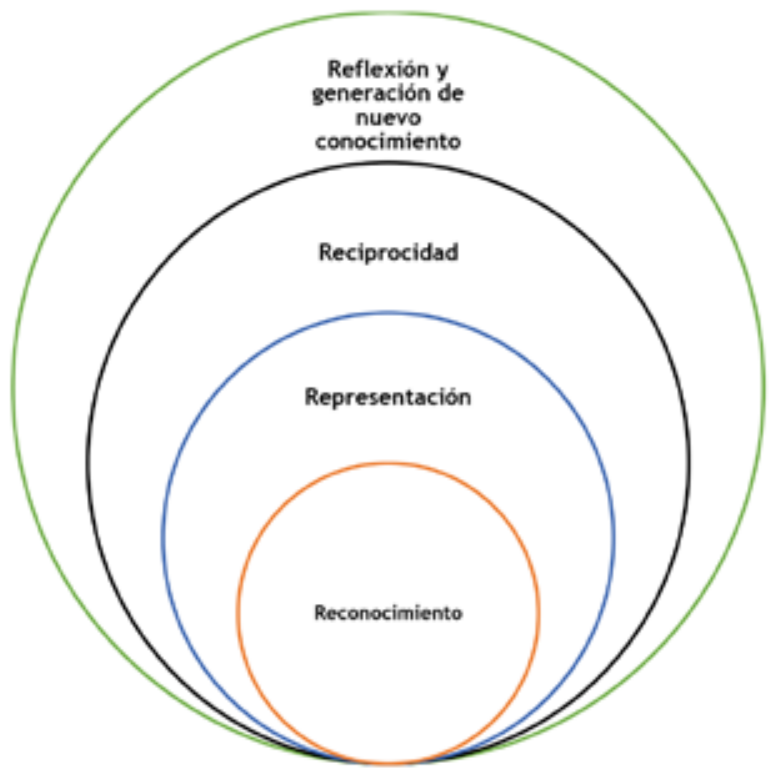

Figura 2: Transcendencia de la Investigación en los CADEP

- Reconocimiento. Se realiza cuando la comunidad tiene la necesidad de explorar las características propias o surgimiento de situaciones problemáticas que no han sido estudiada por la comunidad. ¿Cuál es el problema? ¿Cómo se llama? 
- Representación. Consiste en describir y representar las situaciones, eventos y hechos, es decir, cómo es y cómo se manifiesta un problema en la comunidad. ¿Cuáles son las dimensiones del problema? ¿Qué variaciones existen? ¿Qué es importante acerca del problema?

- Reciprocidad. Su propósito es comprender la relación que existe entre dos o más conceptos, categorías o variables en contexto de una situación problema que la comunidad trata de solucionar. ¿Cuáles son las características del problema? ¿Qué está ocurriendo realmente? ¿Cuál es el proceso por el que surge o se experimenta el problema? ¿Cuáles son los cambios significativos que se están dando? ¿Cuál es la propuesta, alternativa o plan de acción?

- Reflexión y generación de nuevo conocimiento. Se trata de explicar porque ocurre un problema en la comunidad y en qué condiciones se manifiesta o porque se relaciona con otras situaciones del contexto de la comunidad. ¿Cómo actúa el problema? ¿Por qué existe? ¿Cuál es el significado? ¿Cómo se origino el problema? ¿Cuáles son los cambios mas significativos que se están dando?

Los Centros de Apoyo y Desarrollo Educativo Profesional tratan de generar conocimiento en el marco del respeto a la diversidad, la equidad epistémica y el diálogo de saberes. Garantizando la sustentabilidad, la paz, la diversidad cultural, la democracia, la convivencia humana y la comunidad de los pueblos. En este sentido, los CADEP integran una metodología de investigación propia sustentada en la creación y recreación de conocimientos, saberes y prácticas en comunidad.

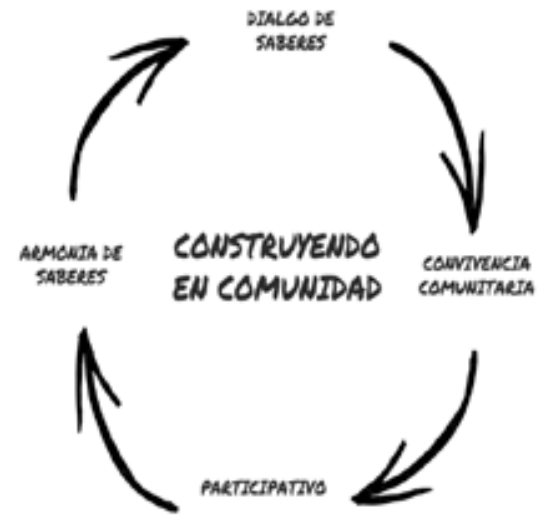

Figura 3: Características de la Investigación en los CADEP

La investigación propia es un proceso que conlleva a desarrollar varios momentos que tejen y articulan la construcción colectiva de la idea, visión y creación, recreación de conocimientos, saberes y prácticas (RUIICAY, 2015): 


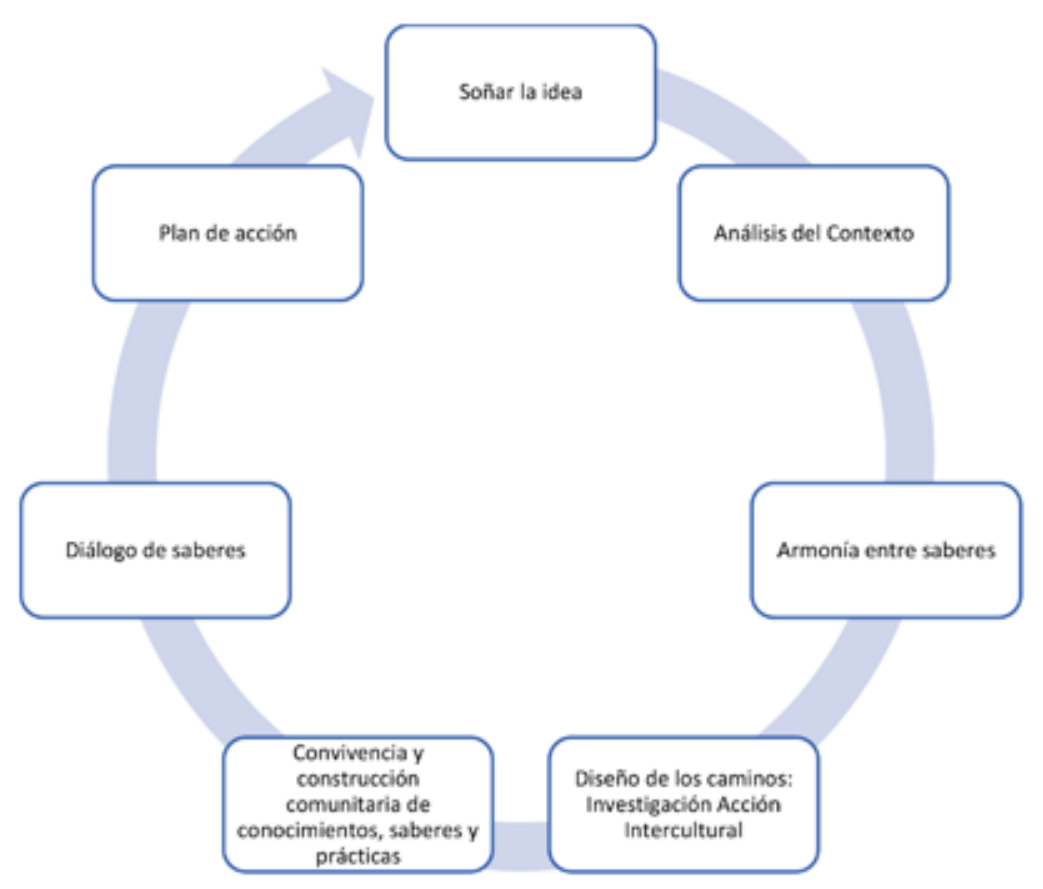

Figura 4: La investigación propia modelo de URACCAN

Soñar la idea. Este primer momento consiste en el consenso comunitario, es decir, entre todos los involucrados en el proceso mediante los consentimientos, previos, libre e informados, definiendo el patrimonio de saberes y conocimientos. Es importante mencionar que deben de partir de la necesidad de la comunidad, de un problema, situación o contexto. En esta etapa se coordinan los espacios y la participación con todos los actores (sujetos-sujetos) del proceso. Análisis del contexto. El análisis de contexto corresponde a todos los elementos integrales de la idea de investigación, en donde es necesario reflejar el estado actual de la temática, entendiéndola de forma integral.

Armonía entre saberes. Es un intercambio de conocimientos, conceptos, saberes, cosmovisiones, espiritualidades, vivencia y práctica sobre la temática seleccionada. Es decir, se fundamenta en la revisión documental, pero también en los saberes orales, incluyendo, además, la orientación epistemológica. Diseño de los caminos: Investigación Acción Intercultural. La investigación acción intercultural es comunitaria para comprender la realidad de los pueblos indígenas, afrodescendientes y comunidades étnicas desde sus propias prácticas y sabidurías ancestrales en diálogo con otros conocimientos. Parte de un diálogo de saberes entre los conocimientos ancestrales y el llamado conocimiento científico o ciencia. Básicamente, pretende contar como se realiza el estudio, destacando las principales técnicas, entre estos diálogos, encuentros de enriquecimientos mutuo, entrevista, grupos focales, observación, talleres e instrumentos y guías, incluyendo de esta forma, la sistematización de la experiencia. 
Convivencia y construcción comunitaria de conocimientos, saberes y prácticas. Es la convivencia con el entorno comunitario que le permite aplicar las técnicas diseñadas y sistematizar los saberes según la idea mutuamente definida por la comunidad. Diálogo de los saberes. Es un compartir entre la información sistematizada a partir de las técnicas y la percepción e interpretación misma de la comunidad sobre ello. Ello implica un proceso de retroalimentación y validación de los conocimientos y sabidurías. Plan de acción. Es el diseño y ejecución de un plan, proyecto o iniciativa diseñada conjuntamente con las comunidades.

\subsection{La Innovación desde los Centros de Apoyo y Desarrollo Educativo Profesional}

Los Centros de Apoyo y Desarrollo Educativo Profesional promueven espacios para el emprendimiento y la innovación, teniendo de referencia que, el emprendimiento es la manera de pensar, sentir y actuar, en la búsqueda de iniciar, crear o formar un proyecto a través de la identificación de ideas y soluciones para el contexto académico (Flores, 2015). Y la innovación es un proceso interactivo activado por la percepción de una oportunidad, proporcionado por un nuevo avance tecnológico que se puede entregar a través de actividades de definición, diseño, producción y compartir de conocimientos. En el ámbito de los CADEP, la innovación es educativa porque contempla diversos aspectos relacionados con la tecnología, didáctica, pedagogía, procesos y comunidad universitaria. La innovación educativa implica la implementación de un cambio significativo en el proceso de enseñanza y aprendizaje.

Desde esta perspectiva, los Centros de Apoyo y Desarrollo Educativo Profesional fomentan un sistema integrado de módulos (Empodera, Innova, Cultiva, Apoya y Convoca) que ofrecen estrategias y recursos que mejoran el ambiente institucional y ofrecen soluciones innovadoras a la comunidad universitaria (ACACIA, 2015), en este sentido, se destacan los tipos de innovación siguientes:

- Innovación en la afectividad social universitaria, mediante sistemas de información capaces de generar recomendaciones didácticas que responden a los estados emocionales del estudiantado que son detectados y seguidos para mejorar su nivel académico y evitar la deserción estudiantil.

- Innovación tecnológica en la docencia universitaria, mediante el uso de sistemas de gestión de conocimiento y soluciones de aprendizaje automático para facilitar el desarrollo de una herramienta de apoyo que detecta las emociones, utilizando entre otro el paradigma de la internet of Things (loT), así como la creación y reutilización de aplicaciones y dispositivos innovadores, fomentando el emprendimiento universitario.

- Innovación en la didáctica en ambientes universitarios mediante: el desarrollo de aplicaciones para apoyar procesos de enseñanza y aprendizajes diferenciados según condiciones cognitivas, afectivas y culturales de los estudiantes, que logran disminuir los niveles de repitencia de asignaturas como la integración de 


\section{REFLEXIONES EDUCATIVAS}

las impresoras $3 \mathrm{D}$ para servir de material educativo de apoyo para estudiantes ciegos; la incorporación de referentes que flexibilizan los currículos universitarios según escenarios y condiciones de aprendizaje de los estudiantes; y ambientes de aprendizaje accesibles para poblaciones con diferencias en el acceso al conocimiento.

- Innovación en la gestión académica universitaria mediante un sistema de gestión de conocimiento para la cooperación solidaria, que detecta fomenta y transfiere innovaciones en lo afectivo, en lo tecnológico y en lo didáctico en sus sistemas de redes de CADEP.

\subsection{Transferencia de Conocimientos desde los Centros de Apoyo y Desarrollo Educativo Profesional}

El Centro de Apoyo y Desarrollo Educativo Profesional se caracteriza por el fomento, fortalecimiento, y transferencia de buenas prácticas que apoyan, cultivan, adaptan, comunican, innovan y acogen a la comunidad universitaria.

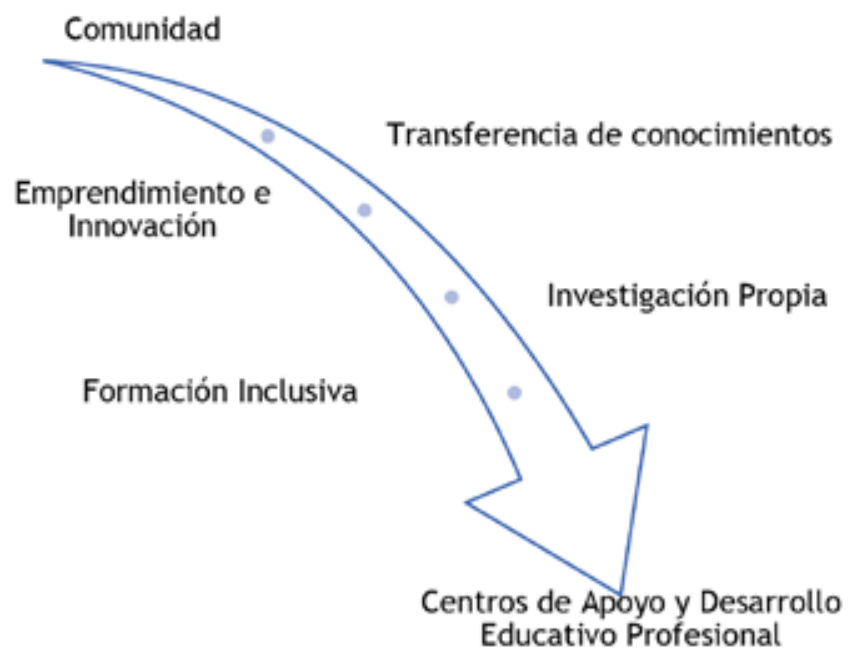

Figura 5: Transferencia de conocimientos, saberes y prácticas

Los CADEP promueven el intercambio y las transferencias de conocimientos y experiencias educativas a partir de la formación, investigación, emprendimiento e innovación. Se trata de promover la co-creación de soluciones para los desafíos de la vida real desde el diseño de la investigación e innovación hasta su puesta en práctica, facilitando así la transferencia de conocimiento, el aprovechamiento de los resultados y el compartir con la comunidad. 


\section{Conclusiones y perspectivas futuras}

Los Centros de Apoyo y Desarrollo Educativo Profesional contribuyen en la desaparición de toda forma de exclusión y discriminación por disparidad o desigualdad, apoyando a quienes se encuentran en desventaja o marginación, incluyendo las necesidades de las personas con discapacidad. Esto implica valorar las instituciones de educación superior como dispositivos sociales y políticos para desarrollar programas de educación multiculturales y plurilingües que respondan a las reales y diversas necesidades educativas; fortalecer la cualificación y capacitación del profesorado; expandir el uso de las tecnologías de la información y comunicación como complemento a los procesos de enseñanza y aprendizaje; y proponer formas novedosas de organización institucional para fomentar la integración de grupos que aúnan esfuerzos y recursos en la solución de problemas relacionados con el contexto de las poblaciones.

Así mismo, los CADEP apoyan, cultivan, adaptan, comunican, innovan y acogen las experiencias, recursos, equipos, problemas, soluciones que requieren las instituciones de educación superior para el fomento profesional de todos sus miembros, para la disminución de la deserción estudiantil, para la modernización de sus estructuras organizativas y para el respeto por el otro y sus diferencias. Esta estructura es una nueva herramienta institucional para detectar, estudiar y solucionar problemas que una institución de educación superior no puede enfrentar de manera aislada (ACACIA, 2015). Entonces, los CADEP, cuentan con un sistema integrado de módulos (empodera, innova, cultiva, apoya y convoca) que cumplen funciones de seguimiento a los estudiantes en riesgo de exclusión social; formación y apoyo para la comunidad universitaria; exploración con sus sistemas de laboratorios de nuevas estrategias para la docencia universitaria y para el uso innovador de las TIC en las prácticas didácticas, estimulando el emprendimiento entre estudiantes y profesores. Este sistema articula la comunidad educativa para un apoyo integral con un enfoque diferenciado del estudiantado.

En definitiva, los Centros de Apoyo y Desarrollo Educativo Profesional promueven la investigación y la innovación didáctica, pedagógica y tecnológica para solucionar problemas en el ámbito universitario, así mismo, diseñan e implementan ambientes de aprendizaje favorables al pensamiento creativo y la interacción entre diversos teniendo de referencia la diversidad de las poblaciones.

\section{Agradecimiento}

A la Unión Europea, específicamente al programa Erasmus Plus, a los Hombres y mujeres profesores e investigadores del proyecto Centros de cooperación para fomento, fortalecimiento y transferencia de buenas prácticas que Apoyan, Cultivan, Adaptan, Comunican, Innovan y Acogen a la Comunidad Universitaria. 


\section{REFLEXIONES EDUCATIVAS}

\section{Lista de referencias}

ACACIA. (2015). Centros de cooperación para fomento, fortalecimiento y transferencia de buenas prácticas que Apoyan, Cultivan, Adaptan, Comunican, Innovan y Acogen a la Comunidad Universitaria. Bogotá: ACACIA.

CEPAL. (2016). Agenda 2030 y los Objetivos de Desarrollo Sostenible: una oportunidad para América Latina y el Caribe. Santiago: CEPAL.

Flores, W., O. (2015). La formación de profesores de matemática desde el ámbito de la Universidad Comunitaria Intercultural. Ciencia e Interculturalidad, 16(1), 32-53. DOI: http://dx.doi.org/10.5377/rci.v16i1.2352

Flores, W., O., Gutiérrez y Restrepo, E., León, O., Sarraipa, J., Pantoja, C., Merino, C., Calderón, D., Guinocchio, M., Rivera, M., Calderón, M., E., \& Boticario, J., G. (2016). Centros de Apoyo y Desarrollo Educativo Profesional para la observación y disminución de la deserción universitaria. Ciencia e Interculturalidad, 18(1), 48-62. DOI: http://dx.doi.org/10.5377/rci.v18i1.3049

Rodríguez, O., León, O. \& Gutiérrez y Restrepo, E. (2017). Fundamentos de los Centros de Apoyo y Desarrollo Educativo Profesional ACACIA. Colombia: Proyecto ACACIA.

RUIICAY. (2015). Cultivo y Crianza de Sabidurías y Conocimientos - CCRISAC. Managua: URACCAN.

UNESCO. (2015). Informe de resultados del Tercer Estudio Regional Comparativo y Explicativo (TERCE). Logros de aprendizaje: laboratorio latinoamericano de evaluación de la calidad de la educación. Santiago: UNESCO.

UNESCO. (2016). Declaración de Incheon y Marco de Acción para la Realización de los Objetivo de Desarrollo Sostenible 4: Educación 2030. Incheon: UNESCO.

UNESCO. (2017). Los nuevos conocimientos emancipatorios desde el sur. Repensado el centenario de la reforma de córdoba y el cincuentenario de mayo de 1968. Quito: UNESCO.

URACCAN. (2017). Política y Agenda Institucional de creación y recreación de conocimientos, saberes y prácticas con perspectiva intercultural de género. Managua: URACCAN.

URACCAN. (2018). Perfil Institucional de la Universidad de las Regiones Autónomas de la Costa Caribe Nicaragüense. Managua: URACCAN. 